\title{
Comparison of State and Parameter Estimators for Electric Vehicle Batteries
}

\author{
R. Morello*, W. Zamboni ${ }^{\S}$, F. Baronti*, R. Di Rienzo*, R. Roncella*, G. Spagnuolo ${ }^{\S}$, and R. Saletti* \\ * Dip. di Ingegneria dell'Informazione, Università di Pisa, Italy \\ $\S$ Dip. di Ingegneria dell'Informazione, Ingegneria Elettrica e Matematica Applicata, Università degli Studi di Salerno, Italy
}

\begin{abstract}
A Battery Management System (BMS) is needed to ensure a safe and effective operation of a Lithium-ion battery, especially in electric vehicle applications. An important function of a BMS is the reliable estimation of the battery state in a wide range of operating conditions. To this end, a BMS often uses an equivalent electrical model of the battery. Such a model is computationally affordable and can reproduce the battery behaviour in an accurate way, assuming that the model parameters are updated with the actual operating condition of the battery, namely its state-of-charge, temperature and ageing state. This paper compares the performance of two battery state and parameter estimation techniques, i.e., the Extended Kalman Filter and the classic Least Squares method in combination with the Mix algorithm. Compared to previous ones, this work focuses on the concurrent estimation of battery state and parameters using experimental data, measured on a Lithium-ion cell subject to a current profile significant for an electric vehicle application.
\end{abstract}

\section{INTRODUCTION}

The Energy Storage System (ESS) is a key element in Electric and Plug-in Hybrid Electric Vehicles (EVs/PHEVs), as well as smart grid systems. In these applications, the Lithium-ion (Li-ion) battery technology is currently the most promising one, thanks to its high energy and power densities, high charge/discharge efficiency and long cycle life. However, Li-ion cells are very sensitive to under discharge, overcharge and to operating temperatures outside the safety range. These conditions can cause permanent damages to the cells and even hazardous situations. To extend the battery life and to avoid dangerous failures, the ESS includes an electronic Battery Management System (BMS). This system mainly monitors current, voltage and temperature of each cell and disconnects the battery from the load/charger when an unsafe condition occurs. BMS also tracks the battery internal state, i.e., the Stateof-Charge $(\mathrm{SoC})$ and the State-of-Health $(\mathrm{SoH})$, to estimate the battery residual energy and to improve its performance through charge balancing strategies [1], [2].

$S o C$ indicates the residual charge that can be provided by a cell at room temperature and at a low ampere-hour rate and is usually expressed in percentage of the cell capacity [3]. As $S o C$ cannot be measured directly, many algorithms have been developed to estimate this important state variable [3]-[10]. Coulomb Counting is the most used technique and is based on current integration. Thus, errors on the current measurement due to current sensor offset, noise and quantisation accumulate and can cause the $S o C$ estimation to become unreliable over time. Another widely used method is based on Open
Circuit Voltage $\left(V_{\mathrm{OC}}\right)$ measurement. $V_{\mathrm{OC}}$ depends on $S o C$, but slightly on temperature, ageing and current rate. However, the cell voltage relaxes to $V_{\mathrm{OC}}$ after a long time, when the cell current is equal to zero. Thus, this technique cannot be used in highly dynamic systems. Other accurate methods are based on a black-box battery model (artificial neural networks and fuzzy logic approaches) [5], but they are computationally intensive and require long training procedures. Good results are reached by the use of model-based algorithms, such as Kalman Filters (KFs) [10], Particle Filters (PFs) [6] and the Mix algorithm [4]. These techniques are closed-loop and suitable for realtime implementation in a BMS. The model is used to predict the cell voltage. The predicted cell voltage is compared with the measured one and the resulting error is used to correct the model state variable estimation. The $S o C$ estimation accuracy thus depends on the model capability to reliably reproduce the cell behaviour.

There are many modelling approaches, such as electrochemical and mathematical models. A good trade-off between complexity and accuracy is given by equivalent electrical models, assuming that their parameters change with the cell operating conditions and ageing. Moreover, also variations in the manufacturing process should be considered. A typical method to account for parameter variation is by means of Look-up Tables (LUTs). However, this solution is suitable only to consider the dependence on temperature and $S o C$ [7]. In fact, online parameter identification is needed to include the ageing and manufacturing variability.

Two promising approaches for battery parameter identification are Moving Window Least Squares (MWLS) method used with the AutoRegressive eXogenous (ARX) structure of the electrical model [8], [11] and Bayesian estimation with the EKF, an extension of the KF for non linear cases [10]. A preliminary comparison of these two methods is presented in [12]. In that work, the battery voltage response is simulated using a LUT-based equivalent cell model [13].

This work significantly extends the analysis in [12], by comparing the capability of these methods to estimate the state and parameters concurrently. Moreover, experimental data representative of the use of the battery in an EV application are used. The MWLS method is used in conjunction with the Mix algorithm to estimate both $\mathrm{SoC}$ and the model parameters. We refer to this method as the Adaptive Mix Algorithm. The paper is organised as follows. Section II recalls the equivalent electrical cell model. Section III describes the compared 


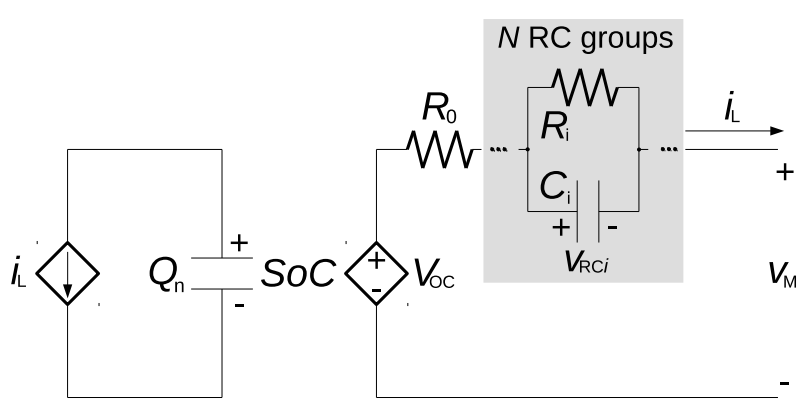

Fig. 1. Equivalent electrical model.

state and parameter estimators, while the experimental results are discussed in Section IV. Finally, Section V draws some conclusions.

\section{EleCtRicAl EQUiVALENT MODEL}

The general form of an equivalent electrical model is shown in Fig. 1. The left-hand side models the cell capacity and $S o C$. In fact, $S o C$ is expressed as $Q / Q_{\mathrm{n}}$, where $Q$ is the remaining charge stored in the cell and $Q_{\mathrm{n}}$ is its maximum value, expressed in Coulomb. Instead, the right-hand side generates the cell terminal voltage $v_{\mathrm{M}}$, as the sum of $V_{\mathrm{OC}}$, a voltage $R_{0} i_{\mathrm{L}}$, due to the internal resistance, and one or more relaxation voltages $v_{\mathrm{RC} i}$. The number of RC groups affects the accuracy of the model and its computational complexity. The transient effects are simulated with accuracy using two RC groups [14]. For applications with fast transients, good results are also achieved using only one $\mathrm{RC}$ group. The consequent low complexity of the model supports its adoption in a BMS. The time-domain state space model of the cell is the following:

$$
\left\{\begin{array}{l}
S \dot{\circ} C=-\frac{i_{\mathrm{L}}}{Q_{\mathrm{n}}} \\
v_{\dot{\mathrm{RC}}}=-\frac{v_{\mathrm{RC}}}{R C}+\frac{i_{\mathrm{L}}}{C} \\
v_{\mathrm{M}}=V_{\mathrm{OC}}-R_{0} i_{\mathrm{L}}-v_{\mathrm{RC}}
\end{array}\right.
$$

Cell model parameters can be extracted offline by observing the voltage/current relationship in the time domain [1]. This method is typically used for cell characterisation, applying a specific current waveform, such as a pulsed profile, to a cell in several conditions. Each pulse has an amplitude and duration, which make the cell $S o C$ vary by a specific amount. Each current pulse is followed by a pause, in which the voltage relaxes towards $V_{\mathrm{OC}}$. The use of a pulsed current facilitates the extraction of the model parameters [14]. The latter can be stored in multi-dimensional LUTs that account for the parameter dependence on $S o C$, temperature and current rate [13].

A LUT-based model is easy to implement, but can hardly model parameter variations due to manufacturing tolerances and ageing. For this reason, an online identification of the cell model parameters should be employed to take into account

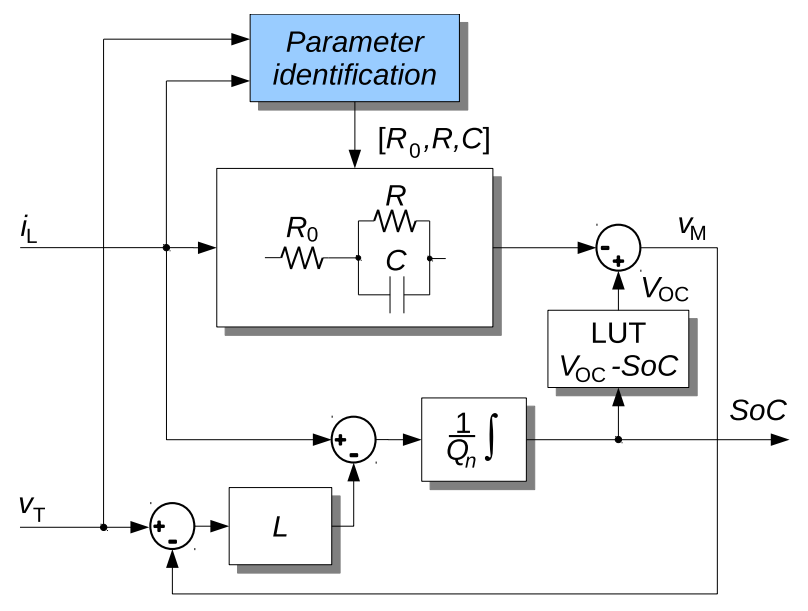

Fig. 2. Adaptive Mix Algorithm block diagram.

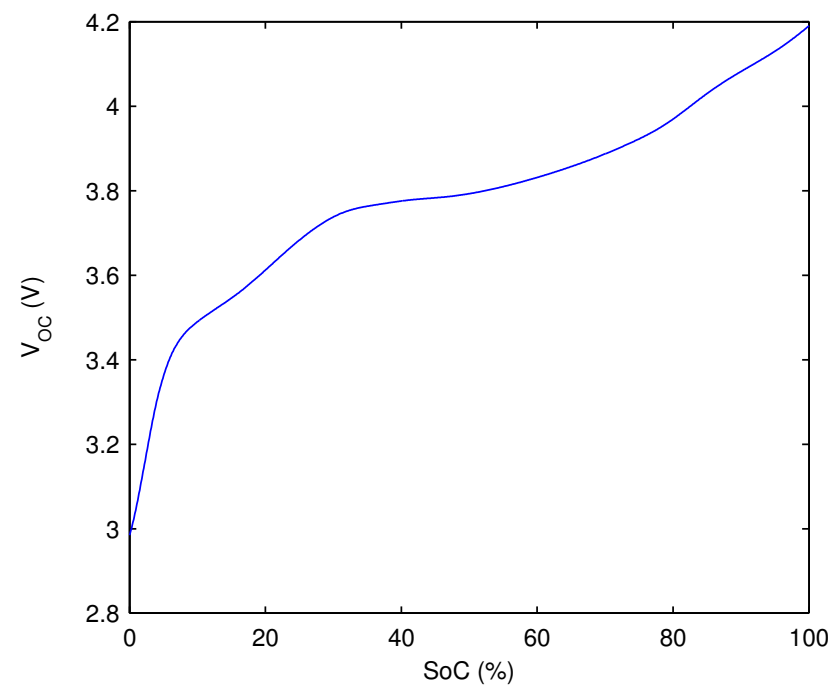

Fig. 3. Open circuit voltage $V_{\mathrm{OC}}$, as a function of $S o C$.

all the operating condition dependencies with the aim of improving $S o C$ estimation in a BMS.

\section{State And Parameter Estimators}

This Section briefly discusses the Adaptive Mix Algorithm (AMA) and the Dual EKF (DEKF), the performance of which will be compared in the subsequent Section.

\section{A. Adaptive Mix Algorithm}

The Mix algorithm is a simple to compute model-based $S o C$ estimation method, firstly introduced in [4]. Its working principle is shown in the block diagram seen in Fig. 2. The model output voltage $v_{\mathrm{M}}$ is generated by the electric model shown in Fig. 1 with a single $\mathrm{RC}$ group. The $V_{\mathrm{OC}}$ voltage is the output of a LUT, which models the $V_{\mathrm{OC}}-S o C$ relationship, shown in Fig. 3, with a $1 \% S o C$ resolution. $v_{\mathrm{M}}$ is compared to the measured cell voltage $v_{\mathrm{T}}$ to generate an error signal. The latter is amplified by the observer gain $L$ and subtracted to the measured cell current $i_{\mathrm{L}}$. The resulting 
current signal is then integrated over time to produce $S o C$, as in the conventional Coulomb Counting method. The observer gain $L$ can be chosen to reduce the sensitivity to uncertainties over the $S o C$ initialisation and the current measurements affecting the Coulomb Counting method [15].

However, such a valuable result relies on the capability of the model to reproduce the cell behaviour in an accurate way. Instead of using three-dimensional LUTs as in [7], a Parameter Identification block is used here to adapt the cell model in order to take into account also parameter variation due to battery ageing and to manufacturing process tolerances. The Parameter Identification block is implemented by the MWLS algorithm, applied to an ARX structure of the electrical model [12], [16].

In more detail, the equivalent electric model is linearised around the time-variant cell operating point and the model parameters are considered constant during the identification time window. This is justified by the fact that the cell operating point slowly changes over time. The $V_{\mathrm{OC}}-S o C$ non-linear function is approximated by a piecewise linear curve $V_{\mathrm{OC}}=$ $\alpha_{0}+\alpha_{1} S o C$, where $\alpha_{0}$ and $\alpha_{1}$ depend on the operating point. Applying the Laplace-transform to state-space cell model (1), we obtain the transfer function from the current input to the cell terminal voltage output:

$$
\frac{Y(s)-\alpha_{0}}{U(s)}=-\frac{R_{0} s^{2}+\left(\frac{\alpha_{1}}{Q_{\mathrm{n}}}+\frac{1}{C}+\frac{R_{0}}{R C}\right) s+\frac{\alpha_{1}}{Q_{\mathrm{n}} R C}}{\left(s+\frac{1}{R C}\right) s}
$$

where $Y(s)$ and $U(s)$ are the Laplace transforms of the voltage output $v_{\mathrm{T}}$ and current input $i_{\mathrm{L}}$, respectively. The discrete-time system transfer function is obtained by the application of the bilinear transform to (2):

$$
\frac{Y\left(z^{-1}\right)-\alpha_{0}}{U\left(z^{-1}\right)}=\frac{b_{0}+b_{1} z^{-1}+b_{2} z^{-2}}{1+a_{1} z^{-1}+a_{2} z^{-2}}
$$

The discrete-time relationship between the input and output samples can be obtain from (3):

$$
\begin{aligned}
y(k)= & -a_{1} y(k-1)-a_{2} y(k-2)+\alpha_{0}\left(1+a_{1}+a_{2}\right) \\
& +b_{0} u(k)+b_{1} u(k-1)+b_{2} u(k-2)
\end{aligned}
$$

which is equivalent to a second order ARX model. The MWLS technique implies the application of the LS method to the current and voltage samples contained into a given time window. This window is shifted in time when new voltage and current samples are acquired, to track the parameter variation over time. In this way, the parameters $\left[a_{1}, a_{2}, b_{0}, b_{1}, b_{2}\right]$ are identified, from which the model parameters $\left[R_{0}, R, C\right]$ can easily be computed [12].

\section{B. Dual EKF}

In the Bayesian framework, the discrete time evolution of the parameters and the system observations are described by the following equations:

$$
\begin{aligned}
p(k+1) & =p(k)+\chi(k), \\
v_{\mathrm{T}}(k) & =\mathcal{G}\left(x(k), i_{\mathrm{L}}(k), p(k)\right)+\psi(k) .
\end{aligned}
$$

Equation (5) is the parameter equation (also called process equation), while (6) is the measurement equation. Here, $k$ is the discrete time, $x$ is the column vector of the system state variables (including $S o C$ ), $i_{\mathrm{L}}$ is the terminal current, $p$ is the column vector of the parameters, $v_{\mathrm{T}}$ is the terminal voltage, $\chi$ and $\psi$ are the parameter and measurement noise, with zero mean and covariance matrix $\Sigma^{\chi}$ and $\Sigma^{\psi}$, respectively. The measurement operator $\mathcal{G}$ is non-linear because of the nonlinear relationship between $V_{\mathrm{OC}}$ and $S o C$ in (1).

Equation (5) is characterised by an identity transition operator acting on the parameters, while the parameter dynamics is caused fictitiously by the Gaussian noise $\chi$. From a practical point of view, this process allows the parameters to change at each time instant, tracking the system dynamically.

In order to perform an online estimation of the parameters, the tracking of the battery state (and in particular of $S o C$ ) is also needed. Indeed, (6) allows the output to be computed from the information on the battery state. The problem can be approached effectively with the so-called dual estimation technique [17], in which two interleaved and interacting processes take place: one for the parameters and the other for the state. This approach can be considered more efficient for online applications, as the involved matrix dimension is lower [9]. The first process is still represented by (5), while the state process equation is given by

$$
x(k+1)=\mathcal{F}\left(x(k), i_{\mathrm{L}}(k), p(k)\right)+\xi(k),
$$

being $\mathcal{F}$ the state operator and $\xi$ the state noise. The measurement equation holding for both state and parameter evolution is (6), which can be used twice, on the basis of the up-to-date values available from the interleaved process.

Given a sequence of observations of $v_{\mathrm{T}}$, the optimal Bayesian estimator, under mild Markovianity hypotheses, can be calculated recursively in two successive steps [17]. For linear Gaussian statistical models, running two interleaved
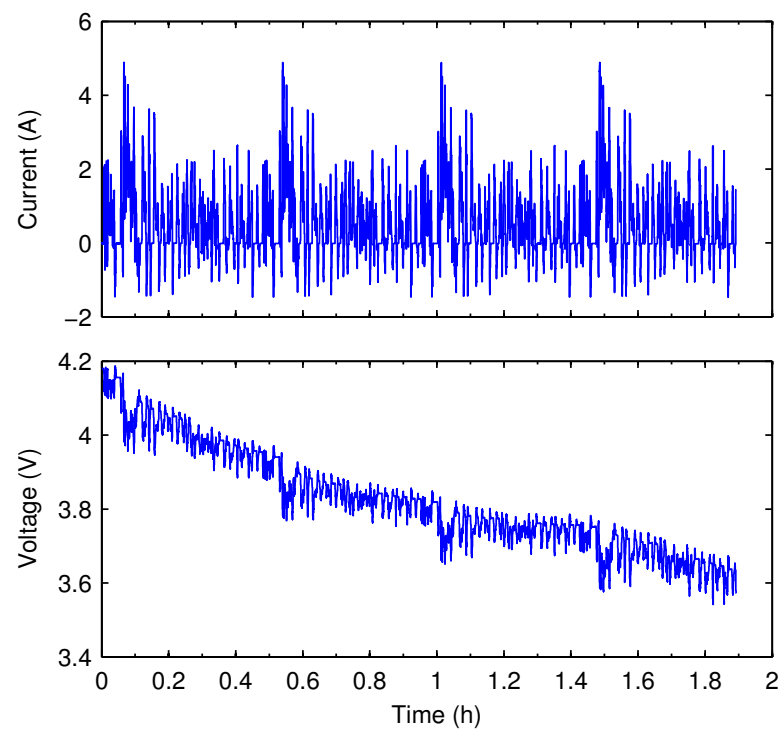

Fig. 4. Cell behaviour in 4 consecutive UDDS cycles. 


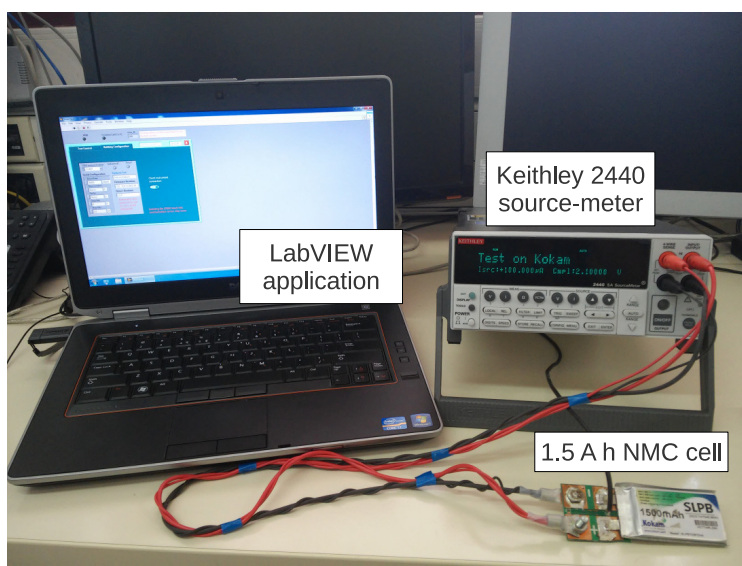

Fig. 5. Photograph of the experimental setup.

Kalman filters yields the optimal Bayesian solution [18]. This method turns out to be very effective also in its extended form, the Extended Kalman Filter, where the presence of non linearities is taken into account [6], [10]. The EKF is based on the application of a linear Kalman filter to the equations, which are linearised around the actual state and parameter estimates. We end up with the DEKF, which has been successfully applied to the state and parameter estimation of batteries, sometimes in combination with other techniques [19]. Implementation details applied to battery state and parameter estimation can be found in [6].

\section{EXPERIMENTAL RESULTS}

Parameter identification strongly depends on the input stimuli. So, a representative battery current profile is necessary to assess the performance of the AMA and the DEKF in an electric vehicle application. To this end, the current profile used to exert the battery in the experimental tests carried out, was derived starting from a standard driving cycle, namely the Urban Dynamometer Driving Schedule (UDDS), defined by the U.S. Environmental Protection Agency [20]. The power at the battery's terminals and then the current is obtained from the speed profile using a simple model of an electric vehicle, as described in [12]. The resulting battery current was scaled maintaining the same C-rate to be applied to a $1.5 \mathrm{~A} \mathrm{~h}$ NMC cell (Kokam SLPB723870H4), used in the experimental tests. The cell has preliminary been characterised by means of pulsed current tests to extract the $V_{\mathrm{OC}}-S o C$ relationship, as shown in Fig. 3, and an average value for the model parameters. The battery current profile related to one UDDS cycle determines approximately a $20 \% S o C$ variation and is repeated 4 times, after a full charge (see Fig. 4). All the tests have been carried out at room temperature.

A photograph of the experimental testbed is shown in Fig. 5. It consists of a Source Meter Unit (SMU) Keithley 2440, controlled by a LabVIEW application running on a PC, which generates the current profile and acquires the cell voltage. The acquired current and voltage samples are processed by Matlab scripts. They implement the AMA and the DEKF, computing the parameters of the cell model and the $S o C$ over time.
Fig. 6 shows the results of the parameter identification, i.e., $R_{0}, R, C$ and $\tau=R C$. The results provided by the two methods are in good agreement with each other and with the average values $\left(R_{0}=26 \mathrm{~m} \Omega, R=12 \mathrm{~m} \Omega\right.$ and $C=780 \mathrm{~F}$ ) extracted from the pulsed current tests. This is especially noticeable for the series resistance $R_{0}$, which is a valuable result, as $R_{0}$ strongly affects the capability of the model to reproduce the cell behaviour and is very sensitive to the cell operating conditions. Indeed, tracking $R_{0}$ over time can provide a good indication of battery ageing, enabling $\mathrm{SoH}$ estimation [21].

To assess the capability of the model with online update of its parameters using the MWLS and the DEKF approaches, the model output is compared to the measured cell voltage. In this analysis, $S o C$ is obtained by the Coulomb Counting of the current measured by the SMU (this value is used as the reference value for the $S o C$ variable). This means that the observer gain $L$ is set to zero for the Mix algorithm. Fig. 7 shows the absolute difference between the measured cell voltage and the voltage predicted by the model (i.e., $\left.e_{\mathrm{v}}=\left|v_{\mathrm{T}}-v_{\mathrm{M}}\right|\right)$, when the parameters are identified by the MWLS method, by the DEKF, and when the parameters are kept constant to their average values. The error behaviour is similar in the three cases, even if a slight improvement can be obtained by the online parameter update, as shown by the maximum and rms values of the voltage prediction error reported in Table I. A greater improvement can be expected when the battery operates at a temperature or ageing state different from that of the characterisation tests.

We now analyse the performance of the concurrent estimation of the battery state and parameters, by comparing the $S o C$ estimated by the AMA, the DEKF and the Mix algorithm (with constant parameters) to the reference value. This is shown in Fig. 8 and in Table II. The estimated $S o C$ behaviours are in very good accordance with the reference one, apart from the $S o C$ interval $50 \%$ down to $25 \%$. In this $S o C$ interval, the $V_{\mathrm{OC}}-S o C$ curve is almost flat (see Fig. 3), thus making $S o C$ poorly observable [15].

Finally, we evaluate the response of the AMA and the DEKF to uncertainty of the initial $S o C$ value. Fig. 9 shows the $S o C$ behaviour estimated by the two methods, when the $S o C$ is initialised to the wrong value of $40 \%$ (instead of the correct $99.3 \%$ ). The slower response of the AMA is determined by the choice of the observer gain $L=1 /\left(R_{0}+R\right)$, which is able to cancel a steady-state error due to an offset in the current sensor [15]. We can see that both algorithms are able to recover from a wrong initialisation, feature that cannot be achieved by the simple Coulomb Counting. Moreover, the DEKF algorithm is much faster than the AMA. In fact, $S o C$ estimated by the DEKF converges to the estimate obtained with the correct initialisation after $40 \mathrm{~s}$ (when the difference between the two cases is lower than $1 \%$ ), whereas this occurs for the AMA after $691 \mathrm{~s}$. 


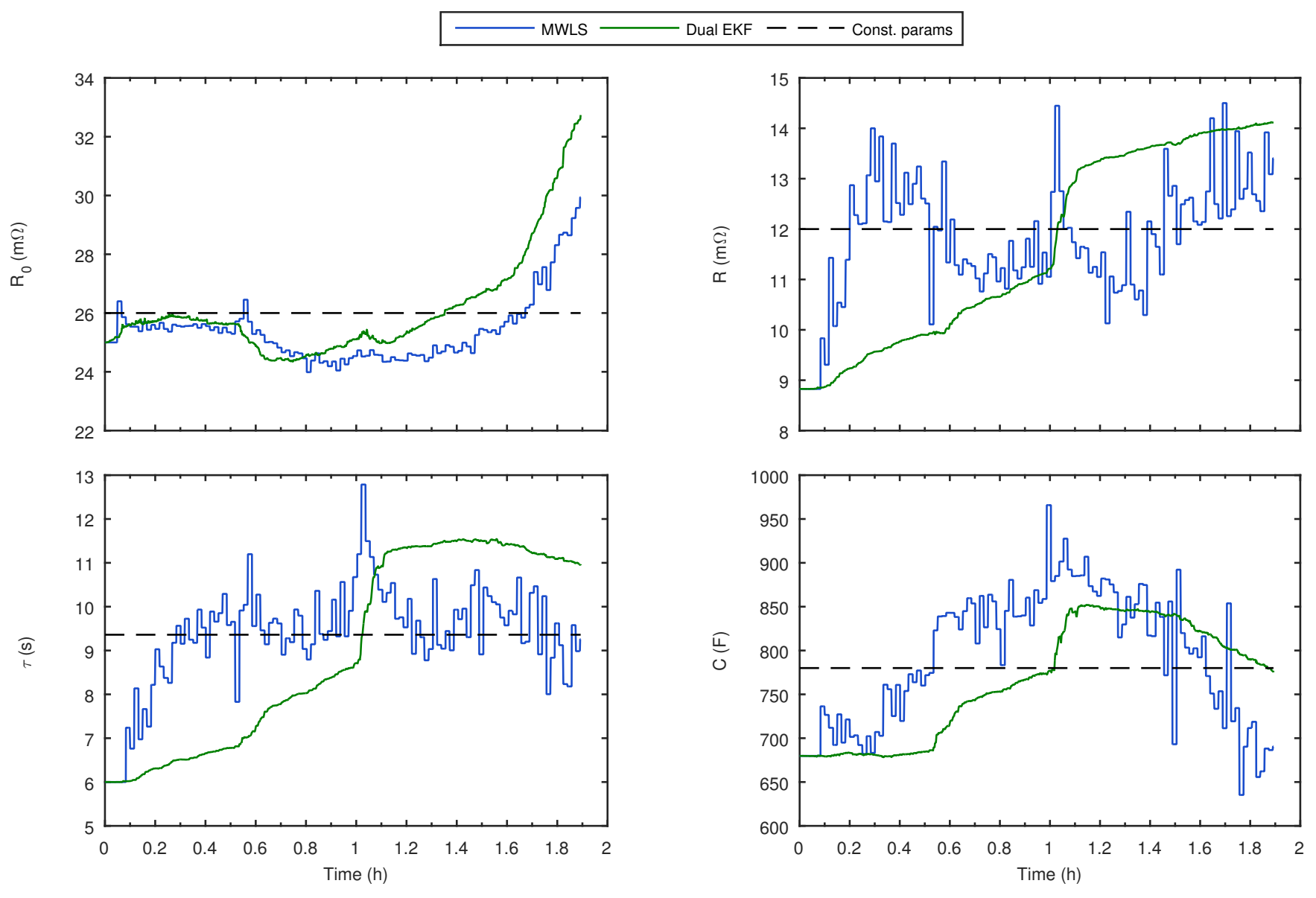

Fig. 6. Comparison of the parameter identification results. The dashed lines (Const. params) indicate the average value of the model parameters extracted from the pulsed current tests.

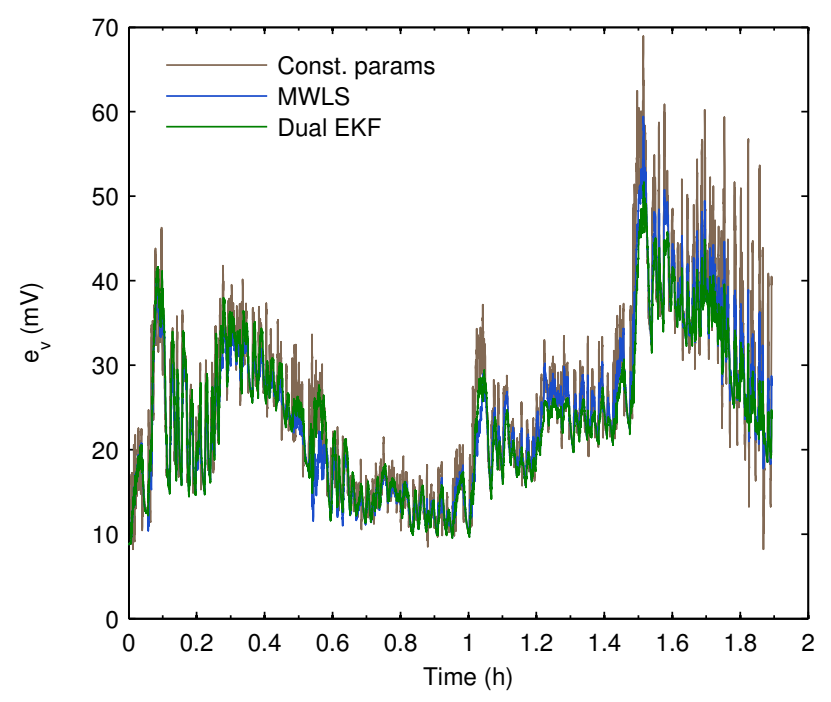

Fig. 7. Comparison of the voltage prediction errors.

\section{CONCLUSION}

This paper has discussed the performance of the Adaptive Mix Algorithm (AMA) and the Dual Extended Kalman Filter (DEKF) to estimate the battery state and parameters in an electric vehicle application. Experimental tests have been
TABLE I

VolTAGE PREDICTION ERROR

\begin{tabular}{lcc}
\hline Estimator & Max & $\mathrm{rms}$ \\
\hline Const. params & $69 \mathrm{mV}$ & $28 \mathrm{mV}$ \\
MWLS & $59 \mathrm{mV}$ & $26 \mathrm{mV}$ \\
Dual EKF & $52 \mathrm{mV}$ & $26 \mathrm{mV}$ \\
\hline
\end{tabular}

performed on a Li-ion NMC cell exerted with a current profile obtained from a standard driving cycle. Both methods provide good estimates of both parameters and $S o C$. The AMA seems to be more noisy in the parameter estimation and shows a slower response to wrong $S o C$ initialisations. On the contrary, it performs slightly better when $S o C$ lies in the range in which the slope of the $V_{\mathrm{OC}}-S o C$ curve is low. This work proves that both methods are capable of identifying the cell model parameters, which is a valuable achievement as the model parameters strongly vary with the cell operating condition.

The real effectiveness of online estimation methods can be appreciated in different operating conditions (temperature and ageing), from those in which the cell characterisation has been executed. For this reason, future work will focus on investigating the algorithm performance in a wider range of operating conditions. Moreover, we will evaluate the computational resources required to implement both methods and, 


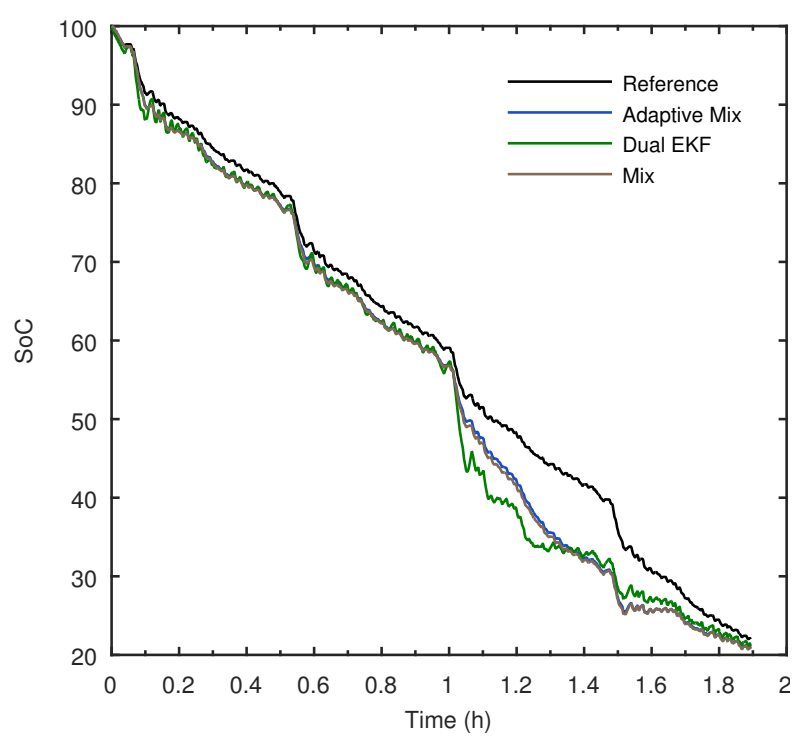

Fig. 8. Comparison of the $S o C$ estimation results.

TABLE II

SOC ESTIMATION ERROR

\begin{tabular}{lcc}
\hline Estimator & Max & rms \\
\hline Adaptive Mix & $9.3 \%$ & $4.3 \%$ \\
Dual EKF & $11.8 \%$ & $5.1 \%$ \\
Mix & $9.7 \%$ & $4.6 \%$ \\
\hline
\end{tabular}

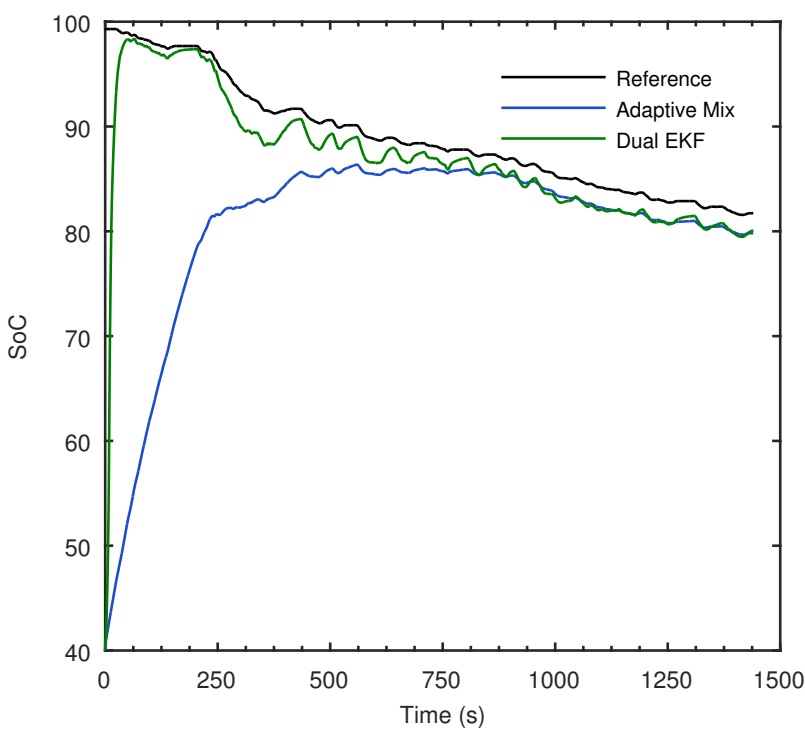

Fig. 9. Comparison of $S o C$ estimation after a wrong initialisation.

thus, their suitability to be used in a BMS for concurrent state and parameter estimation in real time.

\section{REFERENCES}

[1] P. Weicker, A Systems Approach to Lithium-Ion Battery Management. Artech House, 2013.

[2] H. Rahimi-Eichi, U. Ojha, F. Baronti, and M.-Y. Chow, "Battery Management System: An Overview of Its Application in the Smart Grid and Electric Vehicles," IEEE Industrial Electronics Magazine, vol. 7, no. 2, pp. 4-16, Jun. 2013.
[3] S. Piller, M. Perrin, and A. Jossen, "Methods for state-of-charge determination and their applications," Journal of Power Sources, vol. 96, no. 1, pp. 113-120, 2001.

[4] F. Codeca, S. Savaresi, and G. Rizzoni, "On battery state of charge estimation: A new mixed algorithm," in IEEE International Conference on Control Applications, 2008. CCA 2008., Sept 2008, pp. 102-107.

[5] M. Charkhgard and M. Farrokhi, "State-of-charge estimation for Lithium-Ion batteries using neural networks and EKF," IEEE Transactions on Industrial Electronics, vol. 57, no. 12, pp. 4178-4187, Dec 2010.

[6] R. Restaino and W. Zamboni, "Comparing particle filter and extended kalman filter for battery state-of-charge estimation," in IECON 2012 38th Annual Conference on IEEE Industrial Electronics Society, Oct 2012, pp. 4018-4023.

[7] F. Baronti, G. Fantechi, L. Fanucci, E. Leonardi, R. Roncella, R. Saletti, and S. Saponara, "State-of-Charge Estimation Enhancing of Lithium batteries through a Temperature-Dependent Cell Model," in 2011 International Conference on Applied Electronics (AE), Pilsen, 2011. pp. 29-33.

[8] H. Rahimi-Eichi, F. Baronti, and M.-Y. Chow, "Online Adaptive Parameter Identification and State-of-Charge Coestimation for LithiumPolymer Battery Cells," IEEE Transactions on Industrial Electronics, vol. 61, no. 4, pp. 2053-2061, Apr. 2014.

[9] R. Restaino and W. Zamboni, "Rao-blackwellised particle filter for battery state-of-charge and parameters estimation," in IECON 2013 39th Annual Conference of the IEEE Industrial Electronics Society. IEEE, Nov. 2013, pp. 6783-6788.

[10] G. L. Plett, "Extended Kalman filtering for battery management systems of LiPB-based HEV battery packs: Part 3. State and parameter estimation," Journal of Power Sources, vol. 134, no. 2, pp. 277-292, 2004.

[11] H. Rahimi-Eichi, F. Baronti, and M.-Y. Chow, "Modeling and online parameter identification of Li-Polymer battery cells for SOC estimation," in 2012 IEEE International Symposium on Industrial Electronics. IEEE, May 2012, pp. 1336-1341.

[12] F. Baronti, W. Zamboni, N. Femia, H. Rahimi-Eichi, R. Roncella, S. Rosi, R. Saletti, and M.-Y. Chow, "Parameter identification of LiPo batteries in electric vehicles: A comparative study," in 2013 IEEE International Symposium on Industrial Electronics (ISIE), May 2013, pp. $1-7$.

[13] F. Baronti, G. Fantechi, E. Leonardi, R. Roncella, and R. Saletti, "Enhanced model for Lithium-Polymer cells including temperature effects," in IECON 2010 - 36th Annual Conference on IEEE Industrial Electronics Society. IEEE, Nov. 2010, pp. 2329-2333.

[14] M. Chen and G. Rincon-Mora, "Accurate Electrical Battery Model Capable of Predicting Runtime and I-V Performance," IEEE Transactions on Energy Conversion, vol. 21, no. 2, pp. 504-511, Jun. 2006.

[15] F. Baronti, R. Roncella, R. Saletti, and W. Zamboni, "FPGA implemen tation of the mix algorithm for state-of-charge estimation of Lithiumion batteries," in IECON 2014 - 40th Annual Conference of the IEEE Industrial Electronics Society, Oct 2014, pp. 5641-5646.

[16] H. Rahimi-Eichi, F. Baronti, and M.-Y. Chow, "Modeling and online parameter identification of Li-Polymer battery cells for SOC estimation," in 2012 IEEE International Symposium on Industrial Electronics (ISIE), May 2012, pp. 1336-1341.

[17] L. Nelson and E. Stear, "The simultaneous on-line estimation of parameters and states in linear systems," IEEE Transactions on Automatic Control, vol. 21, no. 1, pp. 94-98, Feb. 1976.

[18] R. E. Kalman, "A New Approach to Linear Filtering and Prediction Problems," Transactions of the ASME Journal of Basic Engineering, no. 82 (Series D), pp. 35-45, 1960.

[19] D. Andre, C. Appel, T. Soczka-Guth, and D. U. Sauer, "Advanced mathematical methods of SOC and $\mathrm{SOH}$ estimation for lithium-ion batteries," Journal of Power Sources, vol. 224, pp. 20-27, 2013.

[20] "Urban Dynamometer Driving Schedule (UDDS)." [Online]. Available: http://www.epa.gov/nvfel/testing/dynamometer.htm

[21] D. Haifeng, W. Xuezhe, and S. Zechang, "A new SOH prediction concept for the power lithium-ion battery used on HEVs," in Vehicle Power and Propulsion Conference, 2009. VPPC '09. IEEE, Sept 2009, pp. 1649-1653. 